\title{
Lactarius quieticolor como posible herramienta de control biológico para Fusarium circinatum en planta joven de pino
}

\author{
Otero, M. ${ }^{*}$, Sánchez-Zabala, J. ${ }^{1}$, Martín-Rodrigues, N. ${ }^{1}$, Majada, J. ${ }^{2}$, Txarterina, K. ${ }^{3}$, \\ Azpitarte, J. ${ }^{4}$, Isabel Salcedo, I. ${ }^{1}$, Duñabeitia, M. ${ }^{1}$ \\ ${ }^{1}$ UPV/EHU, Facultad de Ciencia y Tecnología, Departamento de Biología Vegetal y Ecología, \\ Apdo. 644, 48080 Bilbao \\ ${ }^{2}$ Centro Tecnológico Forestal y de la Madera (CETEMAS), Finca La Mata s/n, 33820 Grado, Asturias \\ ${ }^{3}$ BASALAN SA, Avenida Madariaga 1, Dpto. 9, 48014 Bilbao \\ ${ }^{4}$ Confederación de Forestalistas del País Vasco, Barrio Gumuzio S/n, 48960-Galdakao, Bizkaia
}

*Autor para correspondencia: marta.otero@ehu.eus

\begin{abstract}
Resumen
Fusarium circinatum, el hongo patógeno causante del chancro resinoso de los pinos, puede causar graves daños en vivero debido a su capacidad para infectar las raíces de las plántulas y ascender hasta colonizar la parte aérea, causando su muerte en el proceso. Este hecho, unido a que la turba es un hábitat que favorece la supervivencia del inóculo patógeno, insta a buscar nuevas estrategias de manejo de las plantas encaminadas a su control. Con este fin, se ha estudiado el efecto que el hongo simbionte Lactarius quieticolor tiene sobre el crecimiento del patógeno, así como sobre el desarrollo de la infección en planta de Pinus pinaster. El estudio de su potencial como antagonista en condiciones in vitro mostró que en co-cultivo inhibe el crecimiento miceliar del patógeno y, aunque estimula la producción de esporas, inhibe significativamente su germinación tanto en presencia de micelio de L. quieticolor como, en extractos libres del mismo. Asimismo, L. quieticolor incrementó notablemente la tolerancia de la planta a la infección una vez establecido como simbionte en las raíces de plántulas de $P$. pinaster. Los resultados, considerados en conjunto, muestran que L. quieticolor puede ser una herramienta de interés como elemento de control biológico.
\end{abstract}

Palabras clave: biocontrol, ectomicorriza, Pinus pinaster, tolerancia, vivero. 


\section{Introducción}

Fusarium circinatum Nirenberg \& O'Donnell es el hongo causante de "Chancro resinoso" de los pinos, enfermedad considerada como una de las más graves que pueden sufrir estas plantas a nivel mundial por su impacto económico, ecológico y social (Wingfield et al., 2001). Las perdidas más significativas se deben a la reducción del crecimiento de los árboles adultos en las plantaciones forestales y a la muerte masiva de plántulas en vivero (Wingfield et al., 2008). Causa daños en cualquier momento del ciclo vital del pino: floración, semilla, plántula o árbol maduro (Carey et al., 2005), lo que complica mucho su control y manejo. Los síntomas son el amarilleamiento de las acículas, que se secan y caen del árbol, dejando el extremo de la rama desnudo (Gordon et al., 2001) y la aparición de chancros en el tronco (Hepting and Roth, 1946) en fases avanzadas. En planta joven aparece un decaimiento y decoloración de acículas, que finalmente pasan a marrón rojizo, desecación del brote terminal las acículas, desecación del tallo, pudrición de las raíces y muerte de la planta (Wingfield et al., 2008).

En los viveros la aparición de la enfermedad está asociada especialmente a semillas infectadas (Smith et al., 2002; Carey et al., 2005), habiéndose encontrado además, que puede sobrevivir en el suelo (Viljoen et al., 1994) y que es capaz de infectar a las plantas actuando como un patógeno de raíz (Dwinell et al., 1985; MartinRodrigues et al., 2015).

Entre las estrategias de manejo de las plantas encaminadas a reducir la severidad del daño causado, se han ensayado diferentes tratamientos químicos y térmicos. En muchos casos dichos tratamientos no son efectivos y tienen el grave inconveniente de causar fitotoxicidad (Martin-Pinto et al., 2006). Además, también perjudican a la micobiota beneficiosa, lo que a la larga puede resultar perjudicial para la planta al facilitar el posterior establecimiento del patógeno. Por todo ello, la tendencia actual es la de llevar a cabo una producción integrada, que proporcione y mantenga una abundante micobiota (Gordon, 2011). En este campo, las asociaciones ectomicorrícicas pueden jugar un importante papel, ya que fortalecen a la planta y mejoran su desarrollo en condiciones edafoclimaticas desfavorables, que son las que normalmente ocurren en campo (Jones and Hutchinson, 1988). Esto permite a las plantas superar las limitaciones del sitio de plantación (Ortega et al., 2004; Parladé et al., 2004). Además de fortalecer a la planta, la ectomicorriza ejerce una fuerte presión de selección en la comunidad microbiana asociada con la micorrizosfera, lo que contribuye a mejorar su tolerancia al ataque de los patógenos. A los mencionados mecanismos indirectos de protección hay que añadir una protección directa frente a determinados patógenos ejercida por la barrera física que forma la red miceliar del manto (Morin et al., 1999; Sen, 2001), estrategias de antibiosis, competición y/o hiperparasitismo. Tras los trabajos pioneros de Zak (1964) y Marx (1969; 1972), son numerosos los sistemas planta-simbionte-parásito en los que el hongo ectomicorrícico ha demostrado buen potencial como agente de biocontrol, principalmente sobre especies de Phytophtora, Pythium y Fusarium (Whipps, 2004), aunque nunca han sido evaluadas en relación con Fusarium circinatum. 
En este trabajo se parte de la hipótesis de que el hongo ectomicorrícico Lactarius quieticolor Romag. puede ejercer un efecto protector en la planta de Pinus pinaster Ait. frente a la infección por F. circinatum. Por ello, los objetivos planteados son:

- Evaluar la capacidad antagonista de la especie ectomicorrícica L. quieticolor frente a $F$. circinatum in vitro.

- Evaluar el efecto que la micorrización con el hongo ectomicorrícico produce en la respuesta de $P$. pinaster a la infección por el patógeno.

\section{Material y métodos}

\subsection{Evaluación in vitro de la capacidad antagonista de Lactarius quieticolor}

La evaluación de la capacidad antagonista de la especie ectomicorrícica (ECM) Lactarius quieticolor (Lq) se llevo a cabo en placas Petri $(9 \mathrm{~cm} \varnothing)$ sobre medio MMN sólido, utilizando, a nivel de interacción miceliar, la técnica de confrontación de Tang y Lu (1981). Se enfrentó el patógeno con el hongo ECM (Fc-Lq) y el patógeno frente a sí mismo $(\mathrm{Fc}-\mathrm{Fc})$; además, como control, la colonia patógena $(\mathrm{Fc})$ y la ECM (Lq) crecieron enfrentadas a un disco de agar. Tras 45 días en oscuridad y a $25^{\circ} \mathrm{C}$, se determinó el área de crecimiento $\left(\mathrm{cm}^{2}\right)$ con el analizador de imágenes "ImageJ" (Abramoff et al., 2004), el pH del medio y la biomasa (mg) según Eng et al., (2003).

El efecto de L. quieticolor en la capacidad de germinación de las esporas se evaluó en medio solido, en contacto directo con el micelio y en medio liquido, en extractos libres de micelio ECM. En el primer caso se sembraron en torno a 120 esporas por placa $(50 \mu \mathrm{L})$ alrededor de la colonia ECM crecida durante 30 días. Tras incubar 7 días en oscuridad a $25^{\circ} \mathrm{C}$, se midió el $\mathrm{pH}$ del medio y se determino el número de esporas germinadas formadoras de colonias. En el segundo caso, se cultivó $L$. quieticolor en medio MMN líquido durante 15 días y, tras filtrar para eliminar el micelio, analizar el porcentaje de germinación de una concentración final de $10^{6}$ esporas $\mathrm{mL}^{-1}$ en $900 \mu \mathrm{l}$ de filtrado.

\subsection{Evaluación in vivo del efecto de Lactarius quieticolor}

Inicialmente, la semilla de Pinus pinaster se sembró en contenedores CETAP ${ }^{\circledR}$ de 54 alveolos de $200 \mathrm{~cm}^{3}$, sobre mezcla de turba:vermiculita $(1: 1 \mathrm{v} / \mathrm{v})$. La inoculación con Lactarius quieticolor y la caracterización de las plantas se realizó segun Sanchez-Zabala et al., (2013). A los 9 meses, tras la caracterización, se trasplantaron a bandejas QuickPot ${ }^{\mathrm{TM}}$ de 6 alveolos $(110 \mathrm{~mm}$ x $110 \mathrm{~mm}$ x $200 \mathrm{~mm})$ de $1600 \mathrm{~cm}^{3}$ de capacidad, añadiendo a la mezcla de turba:vermiculita $3 \mathrm{~g} \mathrm{~L}^{-1}$ de Plantacote Plus $(14: 18: 15 \mathrm{NPK}+2 \mathrm{Mg})$, para evitar problemas de deficiencias minerales y se dejaron aclimatar durante un mes antes de proceder a la inoculación.

La inoculación consistió en ahoyar ( $3 \mathrm{~cm}$ profundidad y $20 \mathrm{~mm}$ de $\varnothing)$ las cua- 
tro esquinas del contenedor, rellenarlo con inóculo vegetativo patógeno (SanchezZabala, 2013) y taparlo con sustrato. El ensayo constaba de cuatro tratamientos: plantas sin L. quieticolor no inoculadas con el patógeno $(\mathrm{Co})$ y sí inoculadas con el patógeno $(\mathrm{Co}+\mathrm{Fc})$, y plantas micorrizadas con L. quieticolor no inoculadas con el patógeno (Lq) y sí inoculadas con el patógeno $(\mathrm{Lq}+\mathrm{Fc})$. Cada tratamiento constaba de 12 plantas, distribuidas aleatoriamente y mantenidas con riego a demanda durante las 8 semanas que duró el ensayo.

Semanalmente se observó la sintomatología (sanas, marchitez, necrosis, muerte) y se midió el potencial hídrico foliar al mediodía $\left(\Psi_{p o s t}\right)$ mediante cámara de presión (PMS Instrument Company, Model 1000). Al finalizar el ensayo se determinó el porcentaje de supervivencia de las plantas, se analizaron sus sistemas radicales mediante el programa WinRhizo (Vs PRO; ${ }^{\circledR}$ Regent Instruments Inc.) y se trató de reaislar el patógeno sobre medio específico (DCPA agar) a partir de los tejidos del tallo situados $2 \mathrm{~cm}$ por encima del cuello de raíz.

\subsection{Análisis estadístico}

Los datos obtenidos fueron analizados con el paquete estadístico ${ }^{\circ}$ SPSS Inc. (vs. 19.0, 2011). Para determinar las diferencias significativas entre dos grupos o muestras se realizaron análisis T-test; mientras que para más de dos grupos o muestras, se realizó un análisis de varianza $(A N O V A)$ junto con una comparación múltiple post hoc de medias observadas mediante el test SNK. El nivel de significación para los test estadísticos fue $\alpha \leq 0.05$. Los resultados de la evaluación in vitro también fueron evaluados utilizando un análisis de componentes principales (ACP).

\section{Resultados}

\subsection{Evaluación in vitro}

Tras 45 días creciendo en co-cultivo, el dato más relevante proporcionado por las medidas biométricas fue que la presencia de L. quieticolor, inhibió el desarrollo en biomasa de la colonia patógena en un $64.38 \% \pm 1.04$, frente al $39.26 \% \pm 1.31$ causado por la presencia de otra colonia de $F$. circinatum.

Además, aunque la inhibición en área de la colonia del patógeno por parte de $L$. quieticolor $(24.12 \% \pm 3.95)$ no era mayor que la que ejerce el patógeno sobre sí mismo $(27.88 \% \pm 1.85)$, sí que se evidenciaron síntomas de una clara alteración de su morfología (Figura 1). Así se apreció tanto un descenso de la densidad de la colonia, como una llamativa intensificación de la coloración rojiza en la zona de interacción del micelio ECM con el patógeno (Figura 1D), que se correspondía con una degeneración (apoptosis apical) de las hifas patógenas tras su visionado al microscopio. La determinación del pH del medio de cultivo mostró que el valor de 5.5 de inicio descendía a valores de alrededor de 3.5 en todos los casos, sin diferencias significativas entre los diferentes enfrentamientos. 


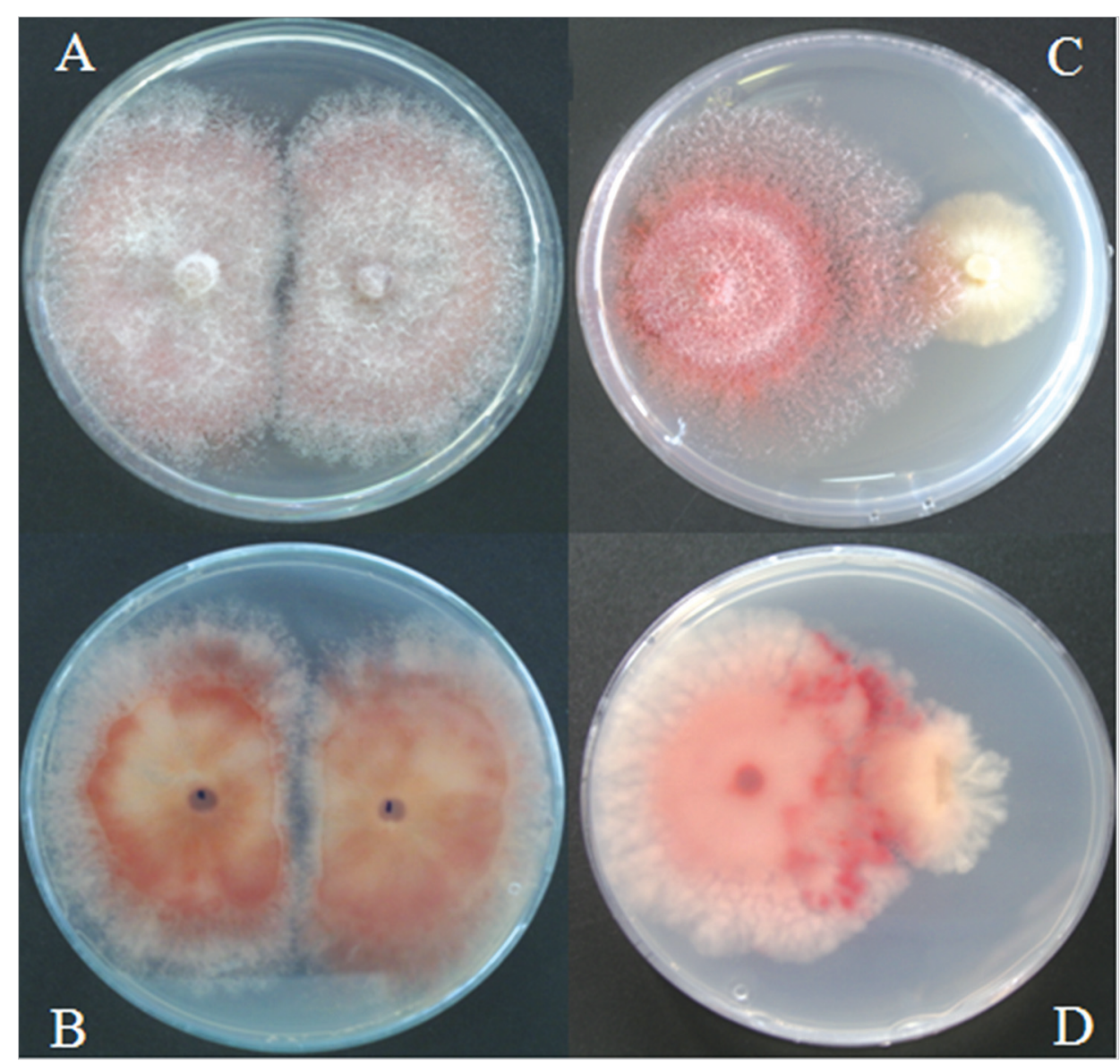

Figura 1. Comparación del aspecto de las colonias de Fusarium circinatum, vistas por el haz (A) y por el envés (B), tras crecer durante 45 días enfrentadas entre sí, con el que presentan cuando crecen enfrentadas a Lactarius quieticolor, caso en el que se ve afectada la densidad de la colonia (C) y la morfología de las hifas, apareciendo, por el envés de las colonias, una llamativa intensificación de la coloración rojiza en la zona de interacción de los micelios (D).

Además de los mencionados cambios en la morfología de la colonia, la presencia de L. quieticolor produjo un significativo incremento de la tasa de producción de esporas (56\%, con respecto a la producción cuando crece enfrentado a si mismo), pasando de una media de $9.35 \cdot 10^{5} \pm 4.81 \cdot 10^{4}$ a $1.45 \cdot 10^{6} \pm 5.42 \cdot 10^{4}$ esporas $\mathrm{cm}^{-2}$. A pesar del incremento en la producción, la germinación de las esporas se vio negativamente afectada tanto en medio sólido, como en extractos de L. quieticolor libres de micelio por efecto de antibiosis. Se obtuvieron valores de inhibición en la tasa de germinación de un $88.88 \% \pm 0.49$ en medio sólido (un 19,72\% de inhibición más que el control) y de un $34.51 \% \pm 3.82$ en el medio líquido en el que previamente había crecido el hongo ECM (un $13.65 \%$ de inhibición más que el control). La determinación del $\mathrm{pH}$ del medio mostró que en el extracto libre de micelio el valor era sólo medio punto más bajo que el del medio control (5.5).

Con objeto de determinar la correlación existente entre las variables estudiadas se realizó un Análisis de Componentes Principales. El resultado fue que las varia- 
bles situadas en el Componente 1 (Inhibición de área, inhibición de biomasa, producción de esporas e inhibición de la germinación) explicaban en un $72 \%$ la varian$\mathrm{za}$, mientras que la variable situada en el Componente $2(\mathrm{pH})$ solo explicaba la varianza en un $20 \%$, de modo que el fenómeno de inhibición ejercido por L. quieticolor sobre F. circinatum sería otorgable al efecto directo que ejerce el primero sobre el segundo, y en menor medida por el efecto indirecto de la alteración del $\mathrm{pH}$.

\subsection{Evaluación in vivo}

El seguimiento de la infección a lo largo del tiempo mostró la aparición de los primeros síntomas a los 28 días de la inoculación con el patógeno, pero solo en las plantas que no estaban micorrizadas con L. quieticolor. Los síntomas iniciales de decaimiento y tristeza progresaron rápidamente a marchitez y finalmente muerte, encontrándose en este estado el $92 \%$ de los pinos a los 49 días de la inoculación del sustrato (Figura 2A). Por el contrario, las plantas micorrizadas con L. quieticolor no evidenciaron ningún síntoma hasta transcurridos 42 días desde la inoculación y, a diferencia de las no micorrizadas, solo un $10 \%$ mostró pérdida de turgencia en acículas y, de este bajísimo porcentaje, solo un $2 \%$ murieron (Figura 2B).

A

$100 \%$

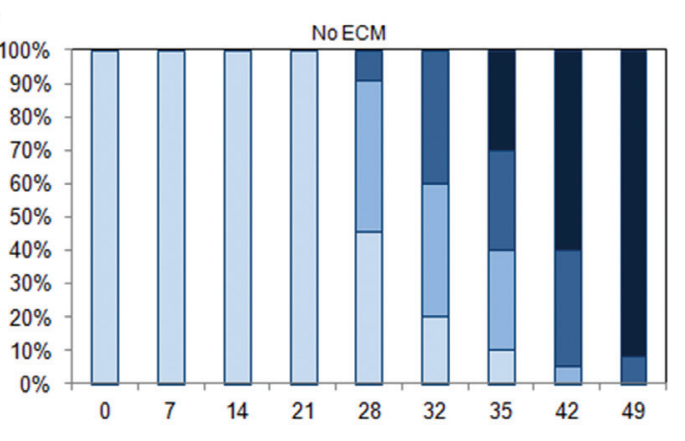

B

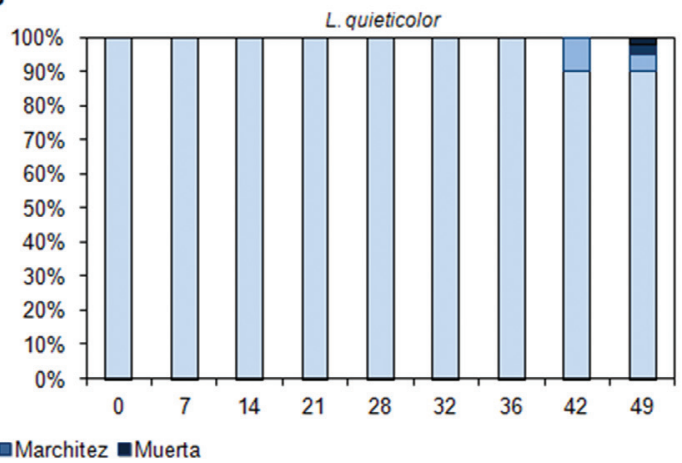

Figura 2. Seguimiento de los síntomas de infección en Pinus pinaster tras inocular su sustrato de crecimiento con Fusarium circinatum. Se ha representado el porcentaje de plantas que no presentan síntomas, así como el porcentaje de las que presentan decaimiento (tristeza), marchitez de acículas o están muertas, en el caso de los pinos no previamente micorrizados (A) y en el de los micorrizados con Lactarius quieticolor (B).

La determinación en el tiempo del potencial hídrico foliar corroboró el estado de alteración de la fisiología de las plantas a partir del día 28, en el caso de las no micorrizadas con L. quieticolor, en las que se produce una caída significativa y progresiva del potencial hídrico alcanzando en la mayoría de los pinos valores medios incompatibles con la vida a partir de la séptima semana (Figura 3). Por el contrario, las plantas micorrizadas con L. quieticolor no muestran variaciones del potencial hídrico hasta transcurridos 42 días y, aun así, el valor medio no bajo de $-1,5$ $\mathrm{MPa}$, lo que indica la ausencia de alteraciones graves de sus relaciones hídricas. 


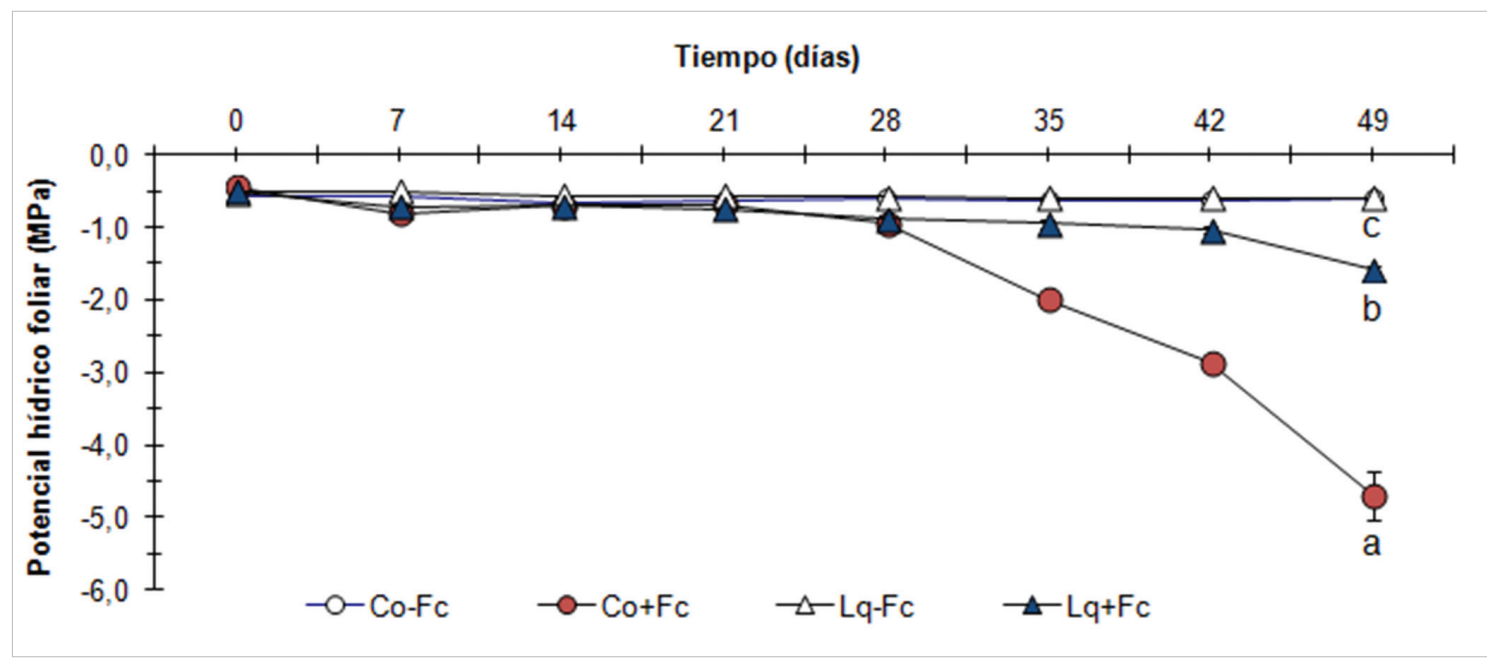

Figura 3. Seguimiento del potencial hídrico foliar a lo largo de los 49 días siguientes a la inoculación del sustrato: pinos $\sin$ Lactarius quieticolor y no inoculados (Co-Fc) o inoculados con Fusarium circinatum $(\mathrm{Co}+\mathrm{Fc})$, y pinos micorrizados con L. quieticolor y no inoculados $(\mathrm{Lq}-\mathrm{Fc})$ o inoculados con el patógeno $(\mathrm{Lq}+\mathrm{Fc})$.

El porcentaje de micorrización de las raíces previo a la inoculación con el patógeno mostró que todas estaban micorrizadas de forma natural (Figura 4A). Así, las plantas no inoculadas con L. quieticolor tenían un $32 \%$ de sus ápices de raíz micorrizados con diferentes especies nativas del vivero. Las plantas inoculadas con la especie ECM, además de tener un $15 \%$ de los ápices de raíz micorrizados con especies nativas del vivero, presentaban un 56\% más de ápices micorrizados con L. quieticolor inoculado. Curiosamente, el intento de reaislamiento del patógeno en medio agarizado a partir de tejidos del tallo obtenidos de zonas situadas $2 \mathrm{~cm}$ por encima del cuello de raíz fue negativo en todas las plantas que estaban micorrizadas con $L$.

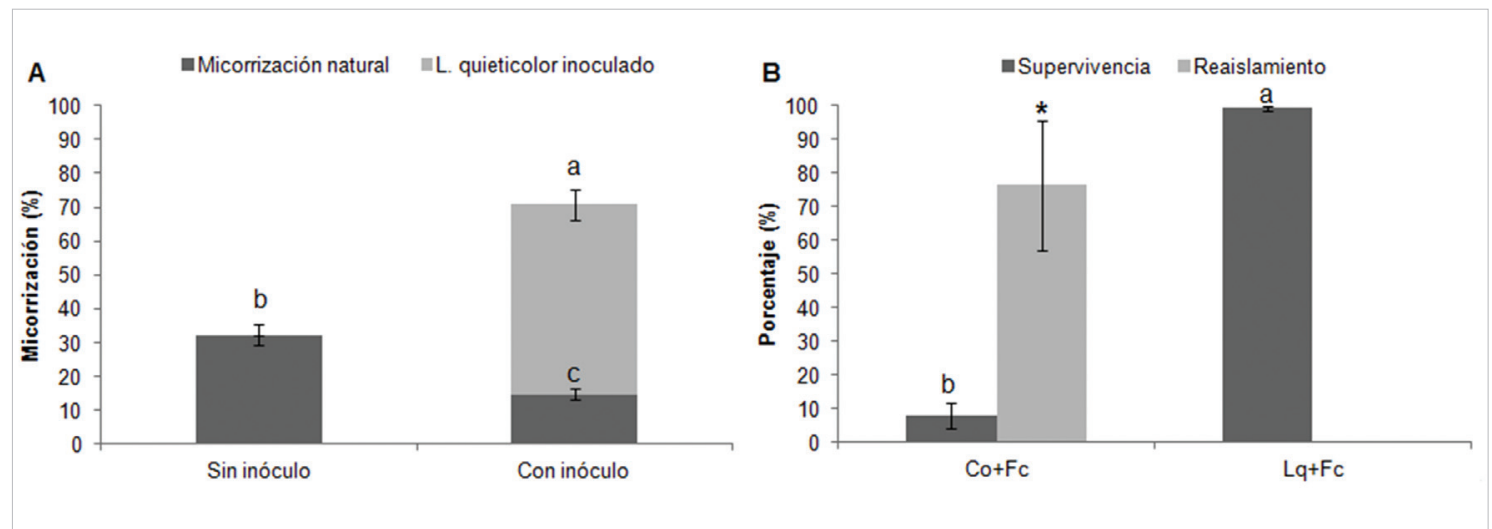

Figura 4. (A) Porcentaje de micorrización ( $\mathrm{n}^{\mathrm{o}}$ de ápices micorrizados respecto al $\mathrm{n}^{\circ}$ total de ápices) que presentaban las plantas de pino inoculadas y no inoculadas con el hongo ECM L. quieticolor en el momento de la inoculación del patógeno en su sustrato de crecimiento. (B) Porcentaje de reaislamientos positivos del patógeno a partir de tejidos del tallo situados a $2 \mathrm{~cm}$ sobre el cuello de raíz. 
quieticolor (Figura $4 B$ ), que a su vez son las que presentaron una tasa de supervivencia de casi el 100\%. Por el contrario, en los pinos no micorrizados con L. quieticolor, y con una tasa de supervivencia de solo un $2 \%$, el patógeno se reaisló en el $80 \%$ de las plantas, lo que indicaba que había conseguido penetrar las raíces y expandirse por la planta hasta alcanzar la parte aérea.

El análisis de la arquitectura radical mostró que en las plantas no micorrizadas con L. quieticolor, la superficie radical (Figura $5 \mathrm{~A}$ ) y el numero de ápices de raíz descendían significativamente (Figura 5B), lo que indica daño en la estructura radical y, en consecuencia, dificultad para mantener una adecuada tasa de absorción de agua. Por el contrario, las plantas micorrizadas con L. quieticolor no presentaban alteraciones de la superficie radical ni del total de ápices de raíz, lo que indicaba ausencia de daño y mantenimiento de la capacidad de absorción de agua y nutrientes.
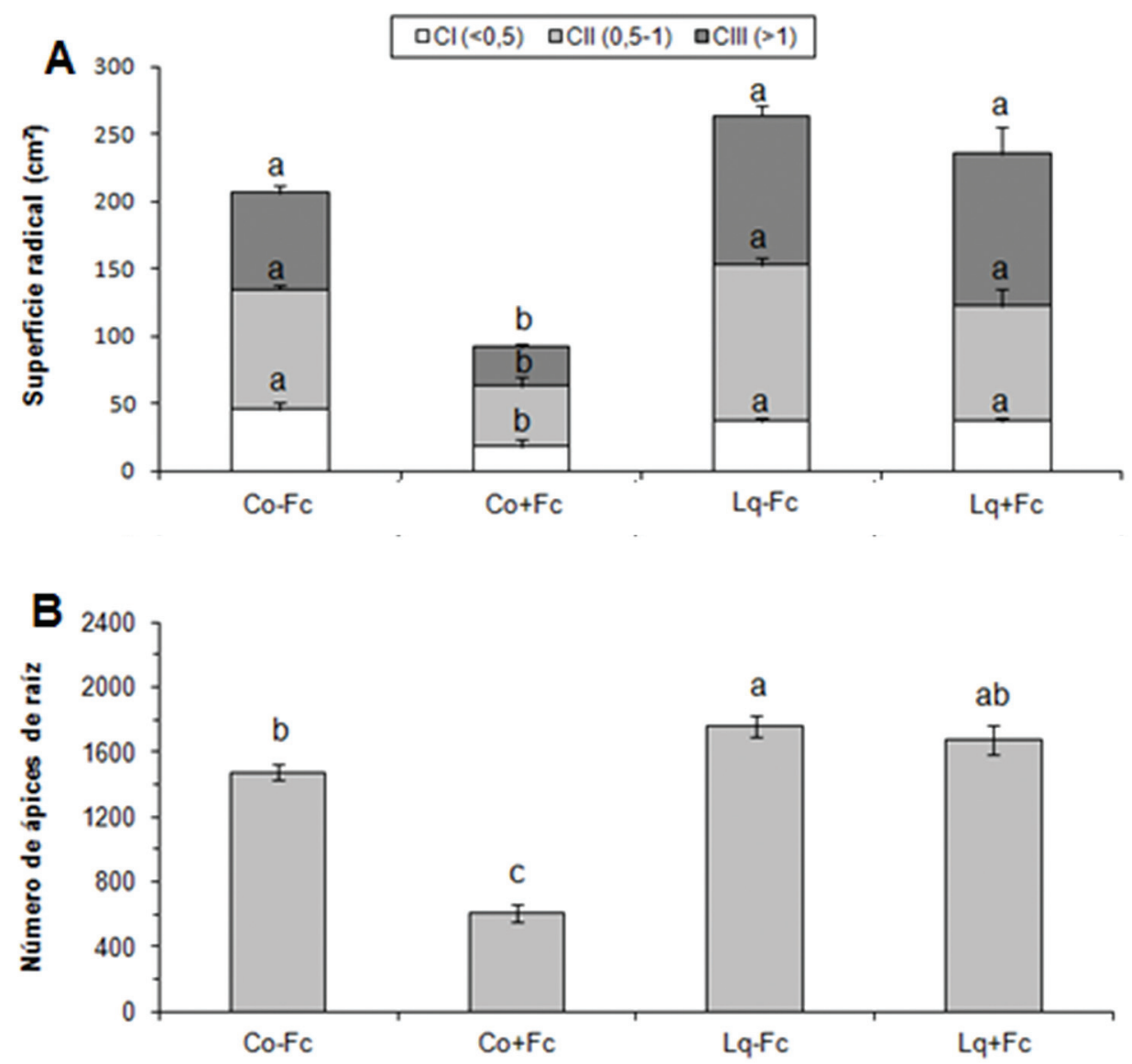

Figura 5. (A) Superficie radical $\left(\mathrm{en}^{\mathrm{cm}} \mathrm{cm}^{2}\right.$ en función del diámetro de las raíces (Clase I: $<0,5 \mathrm{~mm}$; Clase II: 0,5-1 mm; Clase III: > $1 \mathrm{~mm}$ ) y (B) número de ápices de raíz de las plantas de Pinus pinaster no micorrizadas con L. quieticolor y no inoculadas con Fusarium circinatum (Co- $\mathrm{Fc}$ ) o inoculadas con el patógeno $(\mathrm{Co}+\mathrm{Fc})$ y plantas micorrizadas con Lactarius quieticolor no inoculadas $(\mathrm{Lq}-\mathrm{Fc})$ o inoculadas con el patógeno $(\mathrm{Lq}+\mathrm{Fc})$. 


\section{Discusión}

El estudio in vitro de la interacción entre F. circinatum y L. quieticolor muestra la capacidad antagonista de esta especie ECM frente al patógeno. En la bibliografía se pueden encontrar diferentes especies ECM con capacidad para inhibir el crecimiento de especies de Fusarium, como F. oxysporum, F. moliniforme (Martin-Pinto et al., 2006) o F. solani (Zengpu et al., 1989; Vaidya et al., 2005), pero no frente a $F$. circinatum. Nuestro estudio muestra que $L$. quieticolor perjudica el crecimiento de $F$. circinatum, afectando en gran medida a la biomasa y causando una evidente pérdida de densidad de la colonia patógena, además de producir alteraciones morfológicas; destaca especialmente la formación de un frente de color rojo intenso, claramente visible por el envés, en la zona de interacción con el micelio ECM. En este sentido, se han descrito cambios en la morfología de las hifas patógenas que se han atribuido al efecto de metabolitos secundarios con propiedades antifúngicas producidos por los hongos ectomicorrícicos (Kope and Fortin, 1990; Yamaji et al., 2005), que son liberados y difunden por el medio de cultivo (Mucha et al., 2008) con la función de impedir la invasión de su hábitat por competidores potenciales (Sharma et al., 2010).

Asimismo, L. quieticolor es capaz de inhibir la germinación de las esporas patógenas, incluso sin contacto directo, lo que confirma la hipótesis de la implicación de los metabolitos secundarios en la alteración de la fisiología de F circinatum, demostrando que la antibiosis juega un importante papel en la capacidad antágonica de esta especie ECM. Esta observación coincide con lo sugerido por Diez y AlvesSantos (2011) sobre el efecto inhibitorio de extractos obtenidos de cultivos líquidos de Lactarius deliciosus, Rhizopogon roseolus y Tricholoma portentosum en la germinación de las esporas de F. oxysporum y F. verticilloides. También Chakravarty y Hwang (1991) encontraron que los filtrados de cultivos de Laccaria laccata reducían la germinación de las esporas de F. oxysporum y consideraban que se debía a la secreción de compuestos antifúngicos de tipo fenólico. Similares mecanismos de inhibición in vitro han sido postulados por Machón et al. (2006 y 2009) para F. moniliforme, F. oxysporum y F. verticilloides con extractos de cultivos de L. laccata.

Por otra parte, llama la atención que el grado de inhibición del crecimiento del micelio patógeno fuera mayor que el obtenido a nivel de germinación de esporas, lo cual coincide con lo observado por Olaizola (2007), tras evaluar la capacidad de inhibición de extractos líquidos libres de micelio de 21 cepas ECM en la germinación de F. oxysporum y F. verticilloides. Este autor considera que es posible que se deba a que las sustancias inhibitorias producidas en el medio agarificado, o el modo en el que dichas sustancia se difunden por dicho medio sólido, sea cualitativamente o cuantitativamente diferente de los producidos en medio líquido, donde no existe enfrentamiento directo con el patógeno.

Con respecto a la evaluación in vivo, los resultados confirman el efecto antagónico observado in vitro y demuestran que la micorrización con L. quieticolor reduce la severidad del daño y la mortalidad causada por un sustrato infectado con F. circinatum. En ninguna de las plantas colonizadas por L. quieticolor se reaisló el pa- 
tógeno de los tejidos del tallo, ni se observaron daños significativos a nivel de estructura radical ni de sintomatología en parte aérea, manteniendo una tasa de supervivencia de prácticamente el $100 \%$. Por el contrario, en los pinos no micorrizados con L. quieticolor se reaisló de la parte aérea en más de un $80 \%$ de las plantas, lo que junto al muy evidente deterioro de las raíces indica que el patógeno penetró, invadió y se expandió por el sistema radical, colonizando el sistema vascular y desplazándose a la parte aérea, causando en el proceso la muerte del $98 \%$ de las plantas.

La mejora de la nutrición gracias al hongo ECM puede ser considerado un modo de control de la enfermedad (Duchesne, 1994), sin embargo, el efecto protector también está relacionado con interacciones más específicas (Selosse et al., 2004), que actúan directamente al prevenir la infección por el patógeno (Whipps, 2004). Es el caso de la competencia por los nutrientes de la rizosfera y la reabsorción de los exudados radicales por los ápices micorrizados, lo que reduce el atractivo de las raíces para los patógenos (Duchesne et al., 1987a; Sharma et al., 2010). En relación con este mecanismo podrían situarse las diferencias encontradas en el grado de colonización por la flora fúngica nativa del vivero que, en presencia de L. quieticolor baja significativamente y que podría estar relacionada con su competitividad y capacidad antagónica.

Asimismo, entre los mecanismos implicados en la protección directa, el más destacable sería el de la protección física ofrecida por el manto ECM. Se sabe que la presencia de micorrizas de L. laccata y Hebeloma sinapizans en las raíces previene la penetración de Phytophthora cinnamomi (Branzanti et al., 1999). También se han encontrado evidencias claras del papel protector del manto frente a Pythium (Perrin and Garbaye, 1983). En definitiva, la demostrada existencia de una correlación positiva entre el grado de micorrización y el nivel de protección frente al organismo patógeno (Morin et al., 1999) deja claro que la micorrización actúa como una barrera física que dificulta la entrada y el desarrollo del patógeno en el interior de la raíz.

Otro importante mecanismo de protección directa es el de la producción y liberación de compuestos antifúngicos por parte del hongo ECM (Marx and Davey, 1969; Duchesne et al., 1988b) y que, según los resultados de nuestro estudio in vitro, parecen jugar un importante papel en la interacción L.quieticolor-F.ircinatum. Este tipo de compuestos incluyen fenoles y fitoalexinas, así como enzimas que degradan la pared celular del patógeno (Mucha et al., 2006). En este sentido, se ha demostrado que $L$. laccata induce la producción de fenoles en el córtex primario de las raíces protegiéndolas de F. oxysporum en Pseudotsuga menziesii (Sylvia and Sinclair, 1983a) y de F. solani en P. sylvestris (Chakravarty and Unestam, 1987b). Asimismo, se ha encontrado una estimulación de la biosíntesis de terpenos y de la acumulación de compuestos fenólicos en las raíces ectomicorrizadas de plantas jóvenes de diferentes coníferas, cuya acumulación crea una atmósfera fungitóxica en la rizosfera que impide la pudrición de las raíces (Duchesne et al., 1988b).

Los resultados obtenidos sugieren que la inducción de la micorrización con especies seleccionadas podría proteger a las plántulas de pino de la infección por $F$. circinatum, aunque son necesarios más estudios que determinen el nivel de micorriza- 
ción requerido para que resulte eficaz en cada caso. Estos resultados muestran a $L$. quieticolor como un candidato a agente de biocontrol y abren la puerta a la posibilidad de utilizar, en los viveros de planta forestal, determinadas especies ectomicorrícicas como herramienta para proporcionar mayor vigor a la planta y, además, hacerla más tolerante a la infección por $F$. circinatum; con el valor añadido de que, como en este caso, puede tratarse, de especies ECM que producen carpóforos muy demandados desde el punto de vista comercial por su interés gastronómico.

\section{Conclusiones}

Como conclusiones generales del trabajo encontramos que

- L. quieticolor ejerce una capacidad antagónica significativa mediante la inhibición tanto en biomasa, como en la germinación de los conidios de $F$. circinatum.

- Que existen evidencias de que la inoculación de P. pinaster con L. quieticolor en vivero mejora considerablemente la tasa de supervivencia del pino frente a la infección por parte del patógeno, mantiene el sistema radical prácticamente intacto y previene la colonización de las raíces por parte de $F$. circinatum. En vista de los resultados obtenidos en el análisis in vitro se podría considerar que este efecto inhibitorio se debe mayoritariamente a la acción directa por antibiosis del hongo ectomicorrícico y secundariamente al efecto de la bajada de $\mathrm{pH}$ causada por el propio metabolismo fúngico.

\section{Agradecimientos}

Este trabajo ha sido financiado por la Confederación de Forestalistas del País Vasco y la UPV/EHU a través del proyecto Universidad-Empresa US14/04.

\section{Bibliografía}

Abramoff, M.D., Magalhaes, P.J., Ram, S.J., 2004. Image Processing with ImageJ. Biophotonics International. 11, 36-42.

Branzanti, M.B., Rocca, E., Pisi, A., 1999. Effect of ectomycorrhizal fungi on chestnut ink disease. Mycorrhiza. 9, 103-109. doi: 10.1007/s005720050007

Carey, W.A., Oak, S.W., Enebak, S.A., 2005. Pitch canker ratings of longleaf pine clones correlate with Fusarium circinatum infestation of seeds and seedling mortality in containers. Forest Pathol. 35, 205-212. doi: 10.1111/j.1439-0329.2005.00404.x

Chakravarty, P., Unestam, T., 1987b. Differenetial influence of ectomycorrhizae on plant growth and disease resistance in Pinus sylvestris seedlings. J. Phytopathol. 120, 104-120. doi: 10.1111/j.1439-0434.1987.tb04423.x

Chakravarty, P., Hwang, S.F., 1991. Effect of an ectomycorrhizal fungus, Laccaria laccata, 
on Fusarium damping off in Pinus banksiana seedlings. Eur. J. For. Pathol. 21, 97-106. doi: 10.1111/j.1439-0329.1991.tb00949.x

Diez, J., Alves-Santos, F.M., 2011. Use of edible ectomycorrhizal fungi to control Fusarium diseases in forest nurseries. In: Alves-Santos F.M. and Diez J.J. (eds.), Control of Fusarium diseases. Research Signpost, Kerala, India.

Duchesne, L.C., Peterson, R.L., Ellis, B.E., 1987a. The accumulation of plant produced antimicrobial compounds in response to ectomycorrhizal fungi: a review. Phytoprotection. $68,17-27$.

Duchesne, L., 1994. Role of ectomycorrhizal fungi in biocontrol. In: Pfleger FL, Linderman RG (eds.) Mycorrhizae and plant health. The American Phytopathology Society, St. Paul, pp. 27-47.

Duchesne, L.C., Peterson, R.L., Ellis, B.E., 1988b. Pine root exudates stimulate the synthesis of antifungal compounds by the ectomycorrhizal fungus Paxillus involutus. New Phytol. 108, 471-476. doi: 10.1111/j.1469-8137.1988.tb04188.x

Dwinell, L.D., Barrows-Broaddus, J.B., Kuhlman, E.G., 1985. Pitch canker: a disease complex of southern pines. Plant Dis. 69, 270-276. doi: 10.1094/PD-69-270

Eng, F., Gutiérrez-Rojas, M., Favela-Torres, E., 2003. Efecto de la temperatura y el pH en el crecimiento superficial de Botryodiplodia theobromae RC1. Rev. Iberoam. Micol. 20, 172-175.

Gordon, T.R., 2011. Biology and management of Gibberella circinata, the cause of pitch canker in pines. In: Alves_Santos F.M. and Diez J.J. (eds.), Control of Fusarium diseases. Research Signpost, Kerala, India.

Gordon, T.R., Storer, A.J., Wood, D.L., 2001. The Pitch Canker Epidemic in California. Plant Dis. 85, 1128-1139.

Hepting, G.H., Roth, E.R., 1946. Pitch canker, a new disease of some southern pines. J. Forest. 44, 742-744.

Jones, M.D., Hutchinson, T.C., 1988. Nickel toxicity in mycorrhizal birch seedlings infected with Lactarius rufus or Scleroderma flavidum. I. Effects on growth, photosynthesis, respiration and transpiration. New Phytol. 108, 451-459. doi: 10.1111/j.1469-8137.1988. tb04186.x

Kope, H.H., Fortin, J.A., 1990. Antifungal activity in culture filtrates of the ectomycorrhizal fungus Pisolithus tinctorius. Can. J. Bot. 68, 1254-1259. doi: 10.1139/b90-159

Machón, P., Pajares, J.A., Diez, J.J. Alves-Santos, F.M., 2009. Influence of the ectomycorrhizal fungus Laccaria laccata on pre-emergence, post-emergence and late damping-off by Fusarium oxysporum and F. verticillioides on Stone pine seedlings. Symbiosis. 49, 101-109. doi: 10.1007/s13199-009-0015-0

Machón, P., Santamaria, O., Pajares, J.A., Alves-Santos, F.M., Diez, J.J., 2006. Influence of the ectomycorrhizal fungus Laccaria laccata on pre-emergence, post-emergence and late damping-off by Fusarium moniliforme and F.oxysporum on Scots pine seedlings. Symbiosis. 42, 153-160.

Martín-Pinto, P., Vaquerizo, H., Peñalver, F., Olaizola, J., Oria-de-Rueda, J.A., 2006. Early effects of a wildfire on the diversity and production of fungal communities in Mediterranean vegetation types dominated by Cistus ladanifer and Pinus pinaster in Spain For. Ecol. Manage. 225, 296-305. doi: 10.1016/j.foreco.2006.01.006

Martin-Rodrigues, N., Sanchez-Zabala, J., Salcedo, I., Majada, J., Gonzalez-Murua, C., Dunabeitia, M.K., 2015. New insights into radiata pine seedling root infection by Fusarium circinatum. Plant Pathol. doi: 10.1111/ppa.12376 
Marx, D.H., Davey, C.B., 1969. The influence of ectotrophic mycorrhizal fungi on the fungi on the resistance of pine roots to pathogenic infections. IV. Resistance of naturally occurring mycorrhizae to infections by Phytophthora cinnamomi. Phytopathology. 59, 559-565.

Marx, D.H., 1969. The influence of ectotrophic mycorrhizal fungi on the resistance of pine roots to pathogenic infection. I. Antagonism of mycorrhizal fungi to root pathogenic fungi and soil bacteria. Phytopathology. 59, 153-163.

Marx, D.H., 1972. Ectomycorrhizae as biological deterrents to pathogenic root infections. Ann. Rev. Phytopathology. 85, 25-31. doi: 10.090172.002241

Morin, C., Samson, J., Dessureault, M., 1999. Protection of black spruce seedlings against Cylindrocladium root rot with ectomycorrhizal fungi. Can. J. Bot. 77, 169-174. doi: 10.1139/b99-025

Mucha, J., Dahm, H., Strzelczyk, E., Werner, A., 2006. Synthesis of enzymes connected with mycoparasitism by ectomycorrhizal fungi. Arch. Microbiol. 185, 69-77. doi: 10.1007/ s00203-005-0068-2

Mucha, J., Zadworny, M., Werner, A., Napierala-Filipiak, A., Lakomy, P., 2008. Antagonistic activity of the ectomycorrhizal fungus Suillus bovinus challenged by saprotrophic fungi from different soils. Nova Hedwigia. 87, 373-385. doi: 10.1127/0029-5035/2008/ 0087-0373

Olaizola, J., 2007. Selección de hongos ectomicorrícicos comestibles para su utilización en el control biológico del damping-off causado por Fusarium oxysporum Schleht y Fusarium verticillioides Nirenberg. Tesis Doctoral. Universidad de Valladolid.

Ortega, U., Dunabeitia, M., Menéndez, S., Gonzalez-Murua, C., Majada, J., 2004. Effectiveness of mycorrhizal inoculation in the nursery on growth and water relations of Pinus radiata in different water regimes. Tree Physiol. 24, 65-73. doi: 10.1093/treephys/24.1.65

Parlade, J., Pera, J., Luque, J., 2004. Evaluation of mycelial inocula of edible Lactarius species for the production of Pinus pinaster and P. sylvestris mycorrhizal seedlings under greenhouse conditions. Mycorrhiza. 14, 171-176. doi: 10.1007/s00572-003-0252-7

Perrin, R., Garbaye, J., 1983. Influence of ectomycorrhizae on infectivity of Fusarium infested soils and substrates. Plant Soil. 71, 345-351. doi: 10.1007/BF02182674

Sanchez-Zabala, J., 2013. Biotecnología forestal aplicada a la mejora de la producción de Pinus radiata D. Don y Pinus pinaster Ait. Tesis Doctoral. Universidad del País Vasco.

Sanchez-Zabala, J., Majada, J., Martin-Rodrigues, N., Gonzalez-Murua, C., Ortega, U., Alonso-Grana, M., Arana, O., Dunabeitia, M.K., 2013. Physiological aspects underlying the improved outplanting performance of Pinus pinaster Ait. seedlings associated with ectomycorrhizal inoculation. Mycorrhiza. 23, 627-640. doi: 10.1007/s00572-0130500-4

Selosse, M.A., Baudoin, E., Vandenkoornhuyse, P., 2004. Symbiotic microorganisms, a key for ecological succes and protection of plants. C.R. Biologies. 327, 639-648. doi:10.1016/ j.crvi.2003.12.008

Sen, P.R., 2001. Multitrophic interactions between a Rhizoctonia sp. and mycorrhizal fungi affect Scots pine seedlings performance in nursery soil. New Phytol. 152, 543-553. doi: 10.1046/j.0028-646X.2001.00265.x

Sharma, S., Rajak, R.C., Pandey, A.K., 2010. Evidence of antagonistic interactions between rhizosphere and mycorrhizal fungi associated with Dendrocalamus strictus (Bamboo). J. Yeast Fungal Res. 1, 112-117. 
Smith, H., Wingfield, M.J., Coutinho, T.A., 2002. The role of latent Sphaeropsis sapinea infections in post-hail associated die-back of Pinus patula. Forest Ecol. Manag. 164, 177184. doi: 10.1016/S0378-1127(01)00610-7

Sylvia, D.M., Sinclair, W.A., 1983a. Phenolic compounds and resistance to fungal pathogens induced in primary roots of Douglas-fir seedlings by the ectomycorrhizal fungus Laccaria laccata. Phytopathology. 73, 390-397.

Tang, W., Lu, S., 1981. Ecological research methods for plant pathogens. Science Publishing House, Beijing.

Vaidya, G.S., Shrestha, K., Wallander, H., 2005. Antagonistic study of ectomycorrhizal fungi isolated from Baluwa forest (Central Nepal) against with pathogenic fungi and bacteria. Sci. World J. 3, 49-52.

Viljoen, A., Wingfield, M.J., Marasas, W.F.O., 1994. First Report of Fusarium subglutinans f. sp. pini on Pine Seedlings in South Africa. Plant Dis. 78, 309-312. doi: 10.1094/PD78-0309

Whipps, J.M., 2004. Prospects and limitations for mycorrhizas in biocontrol of root pathogens. Can. J. Bot. 82, 1198-1227. doi: 10.1139/b04-082

Wingfield, M. J., Hammerbacher, A., Ganley, R. J., Steenkamp, E. T., Gordon, T. R., Wingfield, B. D., Coutinho, T. A., 2008. Pitch canker caused by Fusarium circinatum - A growing threat to pine plantations and forests worldwide. Australas. Plant Path. 37, 319334. doi: 10.1071/AP08036

Wingfield, M.J., Slipper, B., Roux, J., Wingfield, B.D., 2001. Worldwide movement of exotic forest fungi, especially in the tropics and Southern Hemisphere. BioScience 51, 134-140. doi: 10.1641/0006-3568(2001)051

Yamaji, K.H., Ishimoto, H., Usui, N., Mori, S., 2005. Organic acids and water soluble phenolics produced by Paxillus sp. together show antifungal activity against Pythium vexans under acidic culture conditions. Mycorrhiza. 15, 17-23. doi: 10.1007/s00572-003-0287-9

Zak, B., 1964. Rool of micorrhizae in root disease. Ann. Rev. Phytopathol. 2, 377-392. doi: 10.1146/annurev.py.02.090164.002113

Zengpu, L., Junran, J., Changwen W., 1989. Antagonism between ectomycorrhizal fungi and plant pathogens. Sci. Silvae Sin. 6, 77-81. 